\title{
Comparación de la eficacia de la diálisis entre dos sistemas de control de reinfusión en hemodiafiltración en línea
}

\author{
Sonia Aznar Barbero, Ramón Bel Cegarra, Mª Magdalena Badallo Mira, Bruno David Beltrán Martínez, \\ David Pagan Escribano, Sergio Blázquez Fuentes, Ruth Ponce Palacios, M $^{\mathrm{a}}$ Isabel Beltrán Redondo
}

Enfermeros/as del Servicio de Hemodiálisis del Hospital Santa Lucía. Cartagena. Murcia

\section{Resumen}

La dosis de diálisis y volumen de reinfusión son elementos claves en los mejores resultados comunicados en la hemodiafiltración on-line. Avances tecnológicos permiten dos formas de control automatizado del volumen de reinfusión (una volumétrica y otra por presión), y dos métodos de medición del Kt obtenido por dialisancia iónica (OCM y Diascan). El propósito del presente estudio fue compararlos. Se diseña un estudio prospectivo cruzado sobre población prevalente en hemodiafiltración on-line posdilucional, con consentimiento expreso. Durante 2 semanas consecutivas se realizan tres sesiones con reinfusión automática según hematocrito y proteínas totales, y medición del Kt por OCM, y tres sesiones con reinfusión automática en función de la presión transmembrana y medición del Kt por Diascan. Se compara el valor promedio de volumen de reinfusión y $\mathrm{Kt}$, contrastando las variables con la t-student para muestras relacionadas, estableciendo un valor de significación estadística $p<0,05$. Dieciséis pacientes concluyen el estudio, $56 \%$ hombres, 58,8 años. Un $81,3 \%$ presentan fístula autóloga o protésica como acceso vascular. El volumen de reinfusión es significativamente mayor

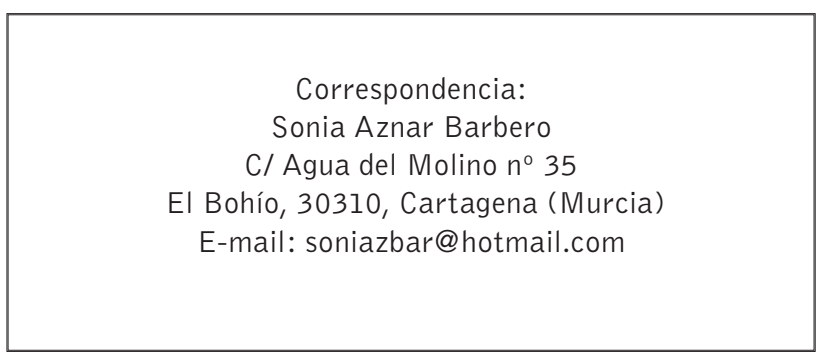

$(p=0,029)$ bajo control de presión $(23,24 \pm 2,55$ I versus $21,81 \pm 1,75 \mathrm{I}$ ) mientras que el $\mathrm{Kt}$ medido por OCM es significativamente $(p<0,001)$ mayor que con Diascan $(59,94 \pm 5,05$ I versus 55,12 $\pm 4,15$ I). Encontramos un incremento medio del volumen de reinfusión $(6,2 \%)$ bajo control de presión, si bien ambos métodos obtienen buenos resultados en los litros de trasporte convectivo final. La dosis de diálisis administrada (Kt) es mayor utilizando OCM.

\section{PALABRAS CLAVE:}

- HEMODIAFILTRACIÓN ON-LINE

- VOLUMEN CONVECTIVO

- KT

\section{Comparison of the dialysis efficacy between two reinfusion control systems in online hae- modiafiltration}

\section{Abstract}

The dose of dialysis and the volume of reinfusión are key elements in the best results communicated in the hemodiafiltration online. Technological advances allow two forms of automated control of reinfusion volume (a volume-based and a pressure-based control system), and two methods of measuring Kt obtained by ionic dialysance (OCM and Diascan). The purpose of this study was to compare both these control systems. We designed a prospective, crossover study of the prevalent population in post-dilution on-line hemodiafiltration, 
with explicit consent. For 2 consecutive weeks, three sessions were performed with automatic reinfusion according to hematocrit and total protein values and Kt measurement with $\mathrm{OCM}$, and three sessions were performed with automatic reinfusion according to transmembrane pressure and Kt measurement with Diascan. The average reinfusion volume and Kt were compared. Variables were contrasted with Student's t-test for related samples. Statistical significance was set at $p<0.05$. Sixteen patients concluded the study ( $56 \%$ men, with a mean age of 58.8 years). A total of $81.3 \%$ had autologous or prosthetic fistulas for vascular access. Reinfusion volume was significantly higher ( $p=0.029)$ under pressure control (23.24 \pm 2.55 Iversus $21.81 \pm 1.75 \mathrm{I}$ ) while Kt measured by OCM was significantly $(p<0.001)$ higher than that measured by Diascan $(59.94 \pm 5.05$ I versus $55.12 \pm$ $4.15 \mathrm{I}$. We found a $6.2 \%$ increase in volume reinfusion under pressure control, although both methods performed well in the final convective transport liters. The dialysis dose (Kt) was higher when OCM was used.

\section{KEY WORDS:}

\section{- ON-LINE HEMODIAFILTRATION}

CONVECTIVE VOLUME

KT

\section{Introducción}

En los últimos años se han descrito diferentes técnicas de hemodiafiltración (HDF) con altos volúmenes convectivos, las cuales constituyen una nueva e interesante aproximación a la forma de depuración del riñón nativo ${ }^{1}$. De entre ellas, la HDF on-line postdilucional es el modo de infusión más eficaz para la eliminación de moléculas de diferentes pesos moleculares ${ }^{2}$.

La magnitud de la convección se ha descrito como esencial en los mejores resultados de la HDFOL en comparación con otras técnicas de hemodiafiltración y hemodiálisis, y posiblemente puede tener relación con la supervivencia del paciente $e^{3,4,5,6}$.

La tecnología permite realizar la reinfusión del líquido de sustitución de forma automatizada, técnica descrita como al menos igual de eficaz que la manual, pero más cómoda y segura ${ }^{7}$. Para ello es preciso conocer el valor de las proteínas totales y hematocrito del paciente. Del mismo modo, el sistema ULTRA permite el control del volumen de reinfusión fijando la presión transmembrana (PTM), con buenos resultados en pre y posdilución ${ }^{8}$.

Por otro lado, la dosis de diálisis es considerada como un elemento fundamental en la diálisis adecuada y en la supervivencia del paciente?. La medición por Kt de la dosis de diálisis ha sido comunicada como más eficaz que mediante el $\mathrm{Kt} / \mathrm{V}^{10}$, ya que este infraestima los casos de diálisis inadecuada, requiere determinaciones analíticas, es manipulable y no medible en cada sesión. Existen dos sistemas de medición del Kt: OCM y Diascan, basados en el método de la dialisancia iónica ${ }^{11}$.

El objetivo del presente estudio prospectivo fue evaluar las diferencias de eficacia de la hemodiafiltración on-line posdilucional medida por Kt y volumen de reinfusión, entre el sistema terapeútico con control volumétrico (monitor $A$ ) y el sistema con control de presión (monitor B). Del mismo modo, valoramos la opinión del personal de enfermería sobre ambos, así como la impresión subjetiva del paciente.

\section{Pacientes y métodos}

Se reclutan 16 pacientes, $56 \%$ hombres, 58,8 años de edad media, 66 meses de permanencia en insuficiencia renal, con etiologías más frecuentes glomerular $(31,3 \%)$, diabética y vascular (25\%), 68,8\% son portadores de fístula nativa, $12,5 \%$ fístula protésica y $18,8 \%$ catéter tunelizado.

Se diseña un estudio prospectivo cruzado sobre población prevalente en HDFOL, todos los pacientes dan su consentimiento expreso. Durante 2 semanas se realizan 6 sesiones consecutivas, de las cuales 3 sesiones con el sistema con control volumétrico (monitor $\mathrm{A}$ ) con reinfusión automática en función de hematocrito y proteínas totales, y medición de $\mathrm{Kt}$ mediante $\mathrm{OCM}$, y otras, 3 sesiones consecutivas con el monitor con control de presión (B) con reinfusión automática en función de la PTM (Ultracontrol) y medición de Kt mediante Diascan. Todas las sesiones se realizan con dializador de poliamida $1,7 \mathrm{~m}^{2}$ de alta permeabilidad, con un tiempo de sesión programado de 240 minutos. 
En cada uno de los periodos se determinan, en valor promedio de las 3 sesiones, flujo sanguíneo (Qb), dosis de diálisis medida por Kt y el volumen total de reinfusión (VTR).

Para medir la opinión que el personal de enfermería y el paciente tienen sobre ambos sistemas de diálisis, se paso una encuesta de valoración subjetiva cuyos principales ítems aparecen en la tabla 1. Todos los ítems se valoran del 1 al 5, siendo 1 lo peor 5 lo mejor.

\section{Encuesta al personal de enfermería $n=16$}

1. Desde el punto de vista ergonómico considera el sistema terapéutico A como: 3,81

\section{2. $Y$ del sistema $B: 3,25$}

3. El interfaz de usuario del monitor A que nos permite la programación de los parámetros on-line lo valora como: 3,88

4. $Y$ el interfaz del monitor $B: 3,88$

5. La instalación del circuito extracorpóreo On-line Plus ${ }^{\circledR}(A)$ le parece: 3,56

6. Y la del circuito Artiset Ultra del monitor B: 4,38

7. Considera la técnica de cebado automatizado del sistema terapéutico $A: 3,19$

8. Y la del monitor $\mathrm{B}: 3,44$

9. Respecto al método conexión/desconexión considera el monitor A vs B como (En esta pregunta, 1 muy difícil, 5 muy fácil): 3,75

10. Valora el modo de reinfusión automatizada en función de Hto y PT del sistema terapéutico A respecto del modo manual tradicional (control-volumen) como: 4,06

11. El modo Ultracontrol del monitor B realiza la reinfusión automática en función de los valores de PTM. ¿Qué le parece esta función respecto del control volumen? 3,94

12. En el sistema On-line $\mathrm{Plus}^{\circledR}(A)$, se puede alcanzar un volumen de sustitución mayor disminuyendo los valores de Hto y PT. ¿Qué le parece esta contingencia? 2,81

13. El sistema terapéutico B permite elevar los valores de PTM consiguiendo un volumen de sustitución mayor, considera esta cuestión como: 3,01

14. La frecuencia de incidencias/alarmas con respecto al supuesto beneficio (mayor $\mathrm{Kt}$ ) una vez modificados estos parámetros en ambos monitores es: 3,13

15. El monitor A mide el Kt real y final mediante el módulo OCM. Su nivel de confianza en éste es: 3,44

16. A su vez el sistema terapéutico B utiliza el Diascan como herramienta para medir el Kt. La efectividad percibida por usted es 2,94

17. En las mismas circunstancias de tratamiento se observan diferencias en el volumen de reinfusión on-line de un monitor a otro, considerando estas como: 3,63

18. Observamos diferencias en cuanto a Kt se refiere de un monitor a otro. Valora estas diferencias como: 3,44
El análisis estadístico se realiza con el programa SPSS 13.0 para Windows. Las variables cualitativas se expresan como frecuencias o porcentajes, y las cuantitativas como media y desviación estándar. Se contrasta normalidad de la muestra mediante test de Kolmogorov. El contraste de hipótesis se reali$z a$, mediante la prueba $t$ de Student. Las variables cualitativas se contrastan con la chi-cuadrado de Pearson. Se establece una significación estadística para $p<0,05$.

\section{Encuesta al paciente $n=16$}

1. Con respecto a la seguridad/confort, considera el monitor $A$ como: 3,43

\section{2. $Y$ el monitor B como: 3,50}

3. La calidad percibida de su diálisis on-line con el monitor A le parece: 3,64

\section{4. $Y$ con el monitor $B: 3,79$}

5. La frecuencia de alarmas con el monitor A la considera: 2,29

6. $Y$ en el monitor $B: 3,57$

7. La dificultad a la hora de que el personal de Enfermería solucione las alarmas/problemas con el monitor A es: 2,07

8. $Y$ con el monitor $B: 3,14$

9. La precisión con la que el monitor A "le quita peso" es: 3

10. Y el monitor B: 3

11. ¿Qué relevancia cree que tiene el monitor $\mathrm{A}$ en la aparición de calambres, hipotensiones, picores...? 2,86

\section{2. ¿Y el monitor $B$ ? 2}

13. El bienestar físico y psicológico cuando se dializa con el monitor A es: 3,57

14. $Y$ con el monitor $B: 3,71$ 


\section{Resultados}

Los resultados del VTR y del Kt se muestran en los gráficos 1 y 2, no apreciándose diferencias significativas en el $\mathrm{Qb}$ entre ambos periodos $(384,19 \pm 12,73$ $\mathrm{ml} / \mathrm{min}$ versus $384,38 \pm 13,17 \mathrm{ml} / \mathrm{min}$ ).

Se aprecian diferencias significativas $(p=0,029)$ en volumen de reinfusión $(23,24 \pm 2,55 \mathrm{I})$ con el sistema de control presión $(B)$ versus $(21,81 \pm 1,75 \mathrm{I})$ el sistema de control volumétrico $(A)$. Los valores de proteínas totales fueron $6,2 \pm 0,4 \mathrm{~g} / \mathrm{dl}$ y de hematocrito de $32,2 \pm 4,1 \%$. PTM inicial de $100 \mathrm{mmHg}$ y reajuste automático, sin superar los $350 \mathrm{mmHg}$.

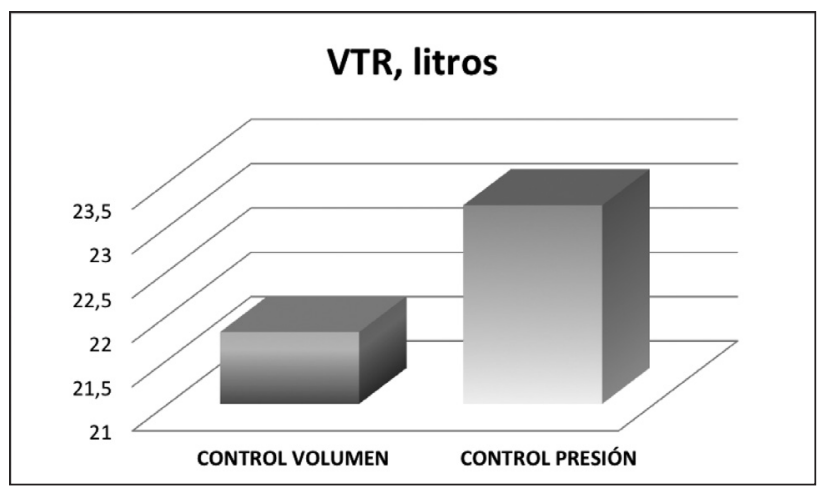

Gráfico 1. Volumen de reinfusión

En cuanto a los resultados del Kt, es significativamente mayor $(p<0,001) 59,94 \pm 5,05$ litros con el sistema A, frente a 55,12 $\pm 4,15$ litros con B. Estas diferencias no se corrigen aplicando la fórmula de Maduell y cols12 (Kt $0 \mathrm{CM}=1,08 \mathrm{Kt}$ Diascan - 2), según la cual Kt0CM esperado sería 57,53 \pm 4,48 litros, inferior $(p<0,001)$ al medido. Los resultados de Kt medido por OCM, Diascan y calculado por la fórmula de Maduell se aprecian en el gráfico 2.

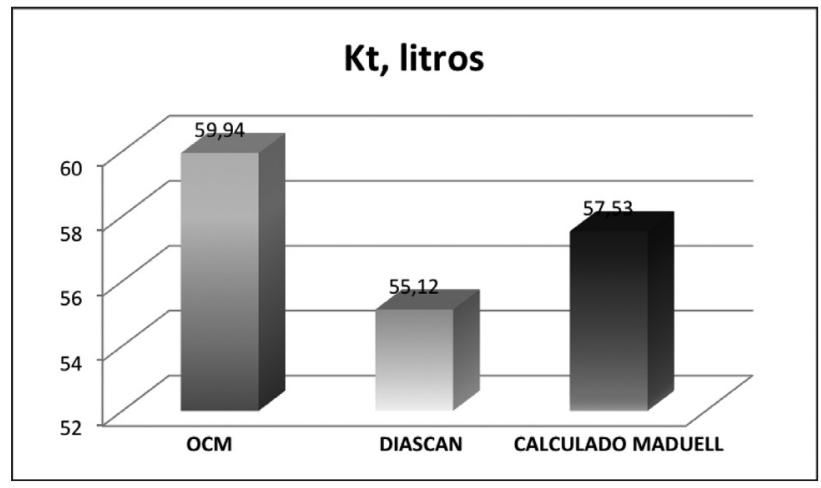

Gráfico 2. Kt
El personal de enfermería valora (tabla 1 ) de forma más importante las diferencias en el VTR $(3,63)$ que en el Kt (3,44, diferencias no significativas). Perciben el Kt más creíble con OCM $(3,44)$ que con Diascan $(2,94$, $\mathrm{p}=0,015)$, valorando muy positivamente la reinfusión automática en ambos monitores (4,06 con el sistema A y 3,94 en B). En general no se muestran muy partidarios de la manipulación de hematocrito-proteinas totales o PTM para alcanzar mejores resultados.

El sistema de control volumétrico es mejor valorado por el enfermero en ergonomía (3,81 versus 3,25 , $p=0,045)$, y en líneas generales se considera más fácil el proceso conexión-desconexión (ítem 9). Del monitor con control de presión se destaca especialmente la instalación del circuito extracorpóreo Artiset $(4,38$ versus $3,56, p=0,003)$ y el sistema de cebado $(3,54$ versus 3,19 , no significativo).

En cuanto al paciente, considera las alarmas en $B$ mayores $(p=0,001)$ que en $A(3,57$ versus 2,29$)$, aunque también afirman que la resolución de las mismas por el personal es más fácil $(3,14$ versus $2,07, p=0,001)$.

En general (tabla 1) B es percibido de forma no significativa como más confortable, segura y de más calidad, atribuyéndole un papel menor en los efectos indeseables de la diálisis ( 2 versus $2,86, p=0,028$ ), y por tanto percibe un mayor bienestar físico y psicológico $(3,71$ versus 3,57, diferencias no significativas).

\section{Discusión}

A la vista de nuestros resultados, el volumen convectivo obtenido bajo control de la PTM es mayor que cuando dicho control es volumétrico. Estos resultados son congruentes con los reportados por Teatini y cols ${ }^{13}$ según los cuales esta mayor eficiencia se debe a una mejor tasa de ultrafiltración, especialmente en pacientes hemoconcentradores con hematocritos altos. Las diferencias han sido aún mayores (cerca de 7 litros) en otra serie ${ }^{14}$.

Los resultados del Kt son igualmente congruentes con los referidos por Maduell y cols ${ }^{12}$, ya que la diferencia real sería de un $10 \%$, un $4 \%$ por la técnica de medición y un $6 \%$ por la depuración de moléculas. En nuestro caso, la distancia real de Kt es de un 8\% (4,82 litros). 
Esta diferencia de eficacia depurativa ha sido explicada por disparidad en el flujo del líquido dializante ${ }^{11}$, si bien los monitores comparados eran otros.

En cuanto a la valoración subjetiva, el personal de enfermería califica bien tanto al sistema A como al monitor B, decantándose un poco hacia el primero, aunque los resultados hay que valorarlos teniendo en cuenta que con este sistema terapéutico se estaba trabajando durante años, mientras que el B estaba recién introducido.

La valoración subjetiva del paciente también es buena para las dos opciones, pero en este caso parecen preferir el monitor con control de presión (B), aunque probablemente también pueden existir interferencias en los resultados por la novedad en nuestra unidad.

\section{Conclusiones:}

Si bien hemos encontrado un incremento medio del volumen de reinfusión $(6,2 \%)$ en las sesiones realizadas con $B$, ambos métodos obtienen buenos resultados en los litros de trasporte convectivo final. La dosis de diálisis administrada medida por Kt es mayor en las sesiones realizadas con A, diferencias que no se explican del todo por los diferentes métodos de medición. Se requieren estudios con mayor tamaño muestral y $\mathrm{Kt} / \mathrm{V}$ analítico para esclarecer dichas diferencias.

Por otro lado, la valoración de ambos monitores ha sido muy buena por el personal de enfermería y por el paciente, que junto a los resultados de eficacia obtenidos, las convierte en dos opciones de última tecnología muy apropiadas para la realización de hemodiafiltración on-line en nuestros pacientes.

Recibido: 13 Julio 2012

Revisado: 14 Agosto 2012

Modificado: 30 Agosto 2012

Aceptado: 31 Agosto 2012

\section{Bibliografía}

1. Maduell F y Arias M. Indicaciones y prescripción de la hemodiafiltración. Dial Trasp 2008: 29(2): 62-66.

2. Maduell F, García H, Hernández-Jara J y cols. Comparación de la infusión predilucional versus postdilucional en la hemodiafiltración en línea. Nefrología 1998; 18; Supl 3-49.

3. Maduell F. Convección versus difusión. ¿Ha llegado el momento del cambio? Nefrología 2009; 29(6):589-593.

4. Alfaro A, Gallego B y cols. HDF en línea en nuestros pacientes: calidad de vida y capacidad funcional. Premio Fresenius Medical Care hemodiafiltración on-line. Rev Soc Esp Enferm Nefrol 2006; 9 (3) 158-163.

5. Canaud B, Braga-Gresham JL, Marshal MR y cols. Mortality risk for patients receiving haemodiafiltration versus haemodialysis: European results from the DOPPS. Kidney Int. 2006; 69: 20872093.

6. Jirka T, Cesare S, Di Benedetto y cols. Mortality risk for patients receiving hemodiafiltration versus hemodialysis. Kidney Int 2006, 70, 1524.

7. Fernández AV, Soto $S$, Arenas $M, y$ cols. Comparación de infusion automatica respecto a manual en hemodiafiltración on line posdilucional. Premio Fresenius Medical Care. Rev Soc Esp Enferm Nefrol 2010;13(1):17-22.

8. Joyeux V, Sijpkens Y, Haddj-Elmrabet A y cols. Optimized convective transport with automated pressure control in on-line postdilution hemodiafiltration. Int J Artific Organs 2008;31(11):928-936.

9. Held PJ, Port FK, Wolfe RA, y cols: The dose of hemodialysis and patients mortality. Kidney Int 1996;50:550-556.

10. Vanesa AV, Soto S, Arenas M, y cols. Estudio comparative de la dosis de diálisis medida pot Kt y Kt/V. Rev Soc Esp Enferm Nefrol 2009;12(2):97-102. 
11. Kulhmann U, Goldau R, Samadi N y cols. Accuracy and safety of online clearance monitoring based on conductivity variation. Nephrol Dial Transplant 2001;16:1053-1058.

12. Maduell $F$, Vera $M$, Arias $M, y$ cols. Influence of the ionic dialysance monitor on Kt measurement in hemodialysis. Am J Kidney Dis 2008;52(1):85-92.

13. Teatini U, Stechiph D, Romei G. Evaluation of a new online haemodiafiltration mode with auto- mates pressure control of convection. Blood Purif 2011:31:259-267.

14. Frouget $T$, Joyeux V, Haddj-Elmrabet A, y cols: Hémodiafiltration en 2005: faut-il continuer à prescrire un volume à infuser ou opter pour la prescription d'une pression transmembranaire constante?. Réunion commune Société francophone de dialyse et Société de néphrologie, Clermont-Ferrand, Septembre 2005 (abstract 31). 